\title{
Changing carriage rate of Neisseria meningitidis among university students during the first week of term: cross sectional study
}

\author{
Keith R Neal, Jonathan S Nguyen-Van-Tam, Nicholas Jeffrey, Richard C B Slack, Richard J Madeley, \\ Kamel Ait-Tahar, Katy Job, Martin C J Wale, Dlawer A A Ala'Aldeen
}

Department of
Public Health
Medicine and
Epidemiology,
University of
Nottingham,
Queen's Medical
Centre, Nottingham
NG7 2UH
Keith R Neal
senior lecturer
Jonathan S
Nguyen-Van-Tam
senior lecturer
Nicholas Jeffrey
medical student
Richard J Madeley
professor
Katy Job
medical student
Meningococcal
Research Group,
Division of
Microbiology,
Queen's Medical
Centre
Richard C Slack
senior lecturer
Kamel Ait-Tahar
PhD student
Dlawer A A
Ala'Aldeen
reader
Communicable
Disease
Surveillance Centre
Trent, Queen's
Medical Centre
Martin C J Wale
regional
epidemiologist
Correspondence to:
K R Neal
keith.neal@
nott.ac.uk
BMJ 2000;320:846-9
Mat

BMJ 2000;320:846-9

website

extra

Techniques for processing the pharyngeal swabs appear on the BMJ's website

www.bmj.com

\begin{abstract}
Objective To determine the rates of, and risk factors for, meningococcal carriage and acquisition among university students.

Design Repeated cross sectional study.

Participants 2507 students in their first year at university.

Main outcome measures Prevalence of carriage of meningococci and risk factors for carriage and acquisition of meningococci.

Results Carriage rates for meningoccoci increased rapidly in the first week of term from $6.9 \%$ on day 1 , to $11.2 \%$ on day 2 , to $19.0 \%$ on day 3 , and to $23.1 \%$ on day 4 . The average carriage rate during the first week of term in October among students living in catered halls was $13.9 \%$. By November this had risen to $31.0 \%$ and in December it had reached $34.2 \%$. Independent associations for acquisition of meningococci in the autumn term were frequency of visits to a hall bar (5-7 visits: odds ratio $2.7,95 \%$ confidence interval 1.5 to 4.8 ), active smoking (1.6, 1.0 to 2.6$)$, being male (1.6, 1.2 to 2.2$)$, visits to night clubs (1.3, 1.0 to 1.6$)$, and intimate kissing (1.4, 1.0 to 1.8$)$. Lower rates of acquisition were found in female only halls $(0.5,0.3$ to 0.9$)$. The most commonly acquired meningococcal strain was C2a P1.5 (P1.2), which has been implicated in clusters of invasive meningococcal disease at other UK universities.
\end{abstract}

Conclusions Carriage rates of meningococci among university students increase rapidly in the first week of term, with further increases during the term. The rapid rate of acquisition may explain the increased risk of invasive meningococcal disease and the timing of cases and outbreaks in university students.

\section{Introduction}

During the 1990s there have been major increases in the incidence of invasive meningococcal disease in many developed countries, ${ }^{1-3}$ with serogroup C disease most noticeable, especially among teenagers and young adults. It has also been shown that university undergraduates have higher rates of invasive meningococcal disease than young adults of the same age who are not attending university. ${ }^{4}$ The provision of places in catered halls seems to be an important factor in differences in rates of invasive meningococcal disease between universities. ${ }^{4}$ In the United Kingdom, several clusters of invasive meningococcal disease have been reported; a large outbreak occurred in November 1996 at the University of Wales in Cardiff 5 and another in October 1997 at the University of Southampton. ${ }^{6}$

No studies have been published on the epidemiology of meningococcal carriage or acquisition among university students in situations where there are no outbreaks. $^{7}$ We therefore performed a longitudinal study in first year university students to determine rates of carriage and acquisition of Neisseria meningitidis, together with risk factors for both.

\section{Participants and methods}

\section{Recruitment of students}

Nottingham University is a large campus based institution. As part of routine induction, all new students (mainly first year undergraduates) are asked to attend the health centre on campus during their first week at university. The order of attendance, evenly distributed across the four days, is set by degree course and not by faculty or hall of residence. During this week in October 1997, we recruited students to the study after they had registered with the health centre and undergone health screening. Each student was given an information sheet and consent form. Those agreeing to take part completed a questionnaire covering: personal characteristics, place of residence, faculty, recent symptoms of upper respiratory tract infection, medical history including meningococcal vaccination, current and recent drugs, travel abroad and to other universities in the past month; active and passive smoking, visits to bars and night clubs, amount and type of alcohol consumed, number of people kissed, and the sharing of glasses and cigarettes in the preceding week. After each student had completed the questionnaire, a trained operator took a posterior pharyngeal swab, which was plated immediately on to selective medium and handled using standard techniques (see website). The same processing methods were used throughout.

\section{Follow up}

All participants in catered halls were selected for a further pharyngeal swab in either the first week of November 1997 or the first week of December 1997 on the basis of odd or even study numbers. Pharyngeal swabs were taken from students in the only self catered hall in the study in December. At the time of reswabbing the questionnaire was repeated. We therefore had paired data available for these students for October and either November or December.

One case of serogroup $\mathrm{C}$ disease occurred in a catered hall in late October, and one third of the students in this hall were therefore given ciprofloxacin to eradicate meningococcal carriage. Although students in this hall were reswabbed according to the prearranged schedule, we excluded them from the main analysis. Two other cases of invasive meningococcal disease (different serogroup B infections) occurred in the study population but these were in the spring term. 


\section{Statistical analysis}

Questionnaire data were scanned with Formic, an electronic scanning package ${ }^{8}$ and stored in Microsoft Access (version 2.0). We used Epi-Info (version 6.04) for $\chi^{2}$ and Fisher's exact tests and spss for Windows (version 8) for multiple logistic regression analysis. Data collected at the time of the first pharyngeal swab were used to determine risk factors for initial carriage through multiple logistic regression. Subsequently, further analyses of risk factors for acquisition during the first term were performed with data from the repeat questionnaires and included only those students whose pharyngeal swab was negative in October.

\section{Results}

Overall, 2507 first year students attended the university's health centre in the first week of the first term, of whom $2453(97.8 \%)$ agreed to participate. A rapid increase in carriage of $N$ meningitidis occurred during the first week (table 1). Date of swabbing, type of hall, active and passive smoking, and intimate kissing were all independent risk factors for meningococcal carriage during the first week (table 2).

In November, 714 of the 939 eligible students $(76.0 \%)$ were reinvestigated for meningococcal carriage and social behaviour. We could not process 172 swabs owing to a problem with an incubator, leaving 542 students (57.7\%). In December, 653 of 933 students $(70.0 \%)$ in catered halls who had participated in the first round were reinvestigated along with 149 of $358(42 \%)$ students in the self catered hall. The figure shows the schedule of swabbing from October to December.

The carriage rate of $N$ meningitidis increased during the first term (table 3). Six weeks after widespread treatment with ciprofloxacin the carriage rate in the excluded hall was 40 of 142 (28.2\%, 95\% confidence interval 20.8 to 35.6). The carriage rate of group C meningococci among students resident in catered halls was $0.5 \%$ (0.2 to 1.0$)$ in October, $1.9 \%$ (0.9 to 3.9$)$ in November, and 3.1\% (2.0 to 4.4) in December.

At some point during the first term, 349 students with initially negative pharyngeal swabs in October acquired meningococci. Table 4 shows the independently significant factors associated with meningococcal acquisition during this period. Overall, 300 of the 325 index strains isolated in October (92\%) and 333 of the 349 strains acquired by students during the autumn term $(95 \%)$ were fully typed. Table 5 shows the full typing of the commonest isolates in the index round and the acquired strains. Although non-C strains predominated on October 1997 (mainly B and non-groupable meningococci), C:2a:P1.5 (P1.2) was the commonest strain acquired (21 of 333) during the first term $\left(\chi^{2}\right.$ $10.8, \mathrm{P}=0.001$.)

\section{Discussion}

Our results show that meningococcal carriage increases rapidly among university students in the first month of the academic year and that much of this increase probably occurs during the first week. Rapid acquisition rates have previously been found among military recruits; however, these studies were generally
Table 1 Carriage rate of Neisseria meningitidis during first week of term, 1997

\begin{tabular}{lcccc} 
& \multicolumn{3}{c}{ No of students } & \\
\cline { 2 - 3 } Date & Swabbed & Positive for $\boldsymbol{N}$ meningitidis & & \% carriage rate $\mathbf{( 9 5 \% ~} \mathbf{C l})$ \\
\hline 30 September & 825 & 57 & $6.9(5.3$ to 8.9$)$ \\
\hline 1 October & 669 & 75 & $11.2(8.9$ to 13.6$)$ \\
\hline 2 October & 691 & 131 & 19.0 (16.0 to 21.9) \\
\hline 3 October & 268 & 62 & $23.1(18.1$ to 28.2) \\
\hline$\chi^{2}$ for linear trend $=74$. & & & \\
$P<0.0001$. & & &
\end{tabular}

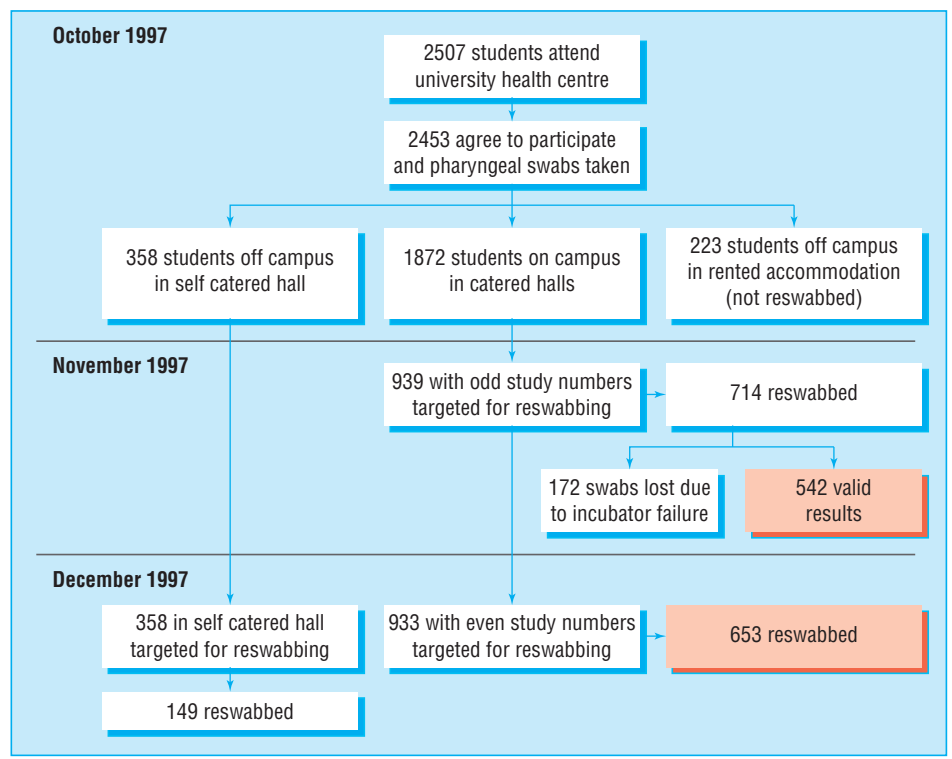

Schedule for pharyngeal swabbing, October to December 1997

smaller and fundamental differences in sleeping arrangements existed compared with students. ${ }^{9-11}$

Several explanations for the rapid increase we observed can probably be discounted. The first was an improvement in swabbing techniques over the first week of the study. Although we were unable to identify the person who took each swab, most were taken by

Table 2 Risk factors for carriage of Neisseria meningitidis during first week of term

\begin{tabular}{|c|c|c|}
\hline Exposure & Odds ratio $(95 \% \mathrm{Cl})$ & $P$ value \\
\hline \multicolumn{3}{|c|}{ Day of throat swab } \\
\hline Tuesday & Reference & \\
\hline Wednesday & 1.74 (1.2 to 2.5$)$ & 0.003 \\
\hline Thursday & 2.99 (2.1 to 4.2 ) & 0.0001 \\
\hline Friday & 4.05 (2.7 to 6.1$)$ & 0.0001 \\
\hline \multicolumn{3}{|l|}{ Type of hall } \\
\hline Mixed sex & Reference & \\
\hline Male only & $1.16(0.8$ to 1.6$)$ & 0.3 \\
\hline Female only & 0.77 (0.5 to 1.3$)$ & 0.4 \\
\hline Off campus & $0.64(0.5$ to 0.9$)$ & 0.01 \\
\hline \multicolumn{3}{|c|}{ Passive smoking (days) } \\
\hline$\overline{0-2}$ & Reference & \\
\hline $3-4$ & 1.26 (0.8 to 1.9$)$ & 0.3 \\
\hline $5-6$ & 1.60 (1.1 to 2.4$)$ & 0.2 \\
\hline 7 & 2.03 (1.3 to 3.1$)$ & 0.001 \\
\hline Smoker & 2.40 (1.6 to 3.7$)$ & 0.0001 \\
\hline \multicolumn{3}{|c|}{ No of people kissed } \\
\hline 0 & Reference & \\
\hline 1 & 1.41 (1.1 to 1.8 ) & 0.01 \\
\hline$\geqslant 2$ & $1.19(0.7$ to 2.0$)$ & 0.5 \\
\hline
\end{tabular}

Each risk adjusted for all other variables in table and antibiotic use in previous month. 
Table 3 Carriage of Neisseria meningitidis by study month and hall status among first year undergraduate university students, 1997

\begin{tabular}{|c|c|c|c|c|}
\hline \multirow[b]{2}{*}{ Month } & \multirow[b]{2}{*}{ Type of hall } & \multicolumn{2}{|r|}{ No of students } & \multirow[b]{2}{*}{$\%$ carriage rate $(95 \% \mathrm{CI})$} \\
\hline & & Swabbed & Positive for $N$ meningitidis & \\
\hline October & Catered & 1872 & 261 & $13.9(12.4$ to 15.1$)$ \\
\hline October & Self catered & 358 & 37 & $10.3(7.2$ to 13.5$)$ \\
\hline November & Catered $^{\star}$ & 542 & 168 & 31.0 (27.1 to 34.9$)$ \\
\hline December & Catered $^{*}$ & 653 & 223 & 34.2 (30.5 to 37.8$)$ \\
\hline December & Catered $\dagger$ & 142 & 40 & 28.2 (20.8 to 35.6$)$ \\
\hline December & Self catered & 149 & 36 & 24.2 (17.3 to 31.0$)$ \\
\hline
\end{tabular}

${ }^{*}$ Excludes results where $30 \%$ of students received ciprofloxacin.

$+30 \%$ of students given ciprofloxacin in late October.

Table 4 Risk factors for acquisition of Neisseria meningitidis during first term

\begin{tabular}{|c|c|c|}
\hline Exposure & Odds ratio $(95 \% \mathrm{Cl})$ & $P$ value \\
\hline \multicolumn{3}{|l|}{ Sex } \\
\hline Female & Reference & \\
\hline Male & 1.61 (1.2 to 2.2$)$ & 0.003 \\
\hline \multicolumn{3}{|c|}{ Passive smoking (days) } \\
\hline $0-2$ & Reference & \\
\hline $3-7$ & $1.21(0.9$ to 1.7$)$ & 0.2 \\
\hline Smoker & $1.6(1.0$ to 2.6$)$ & 0.05 \\
\hline \multicolumn{3}{|c|}{ Weekly visits to hall bar } \\
\hline 0 & Reference & \\
\hline $1-4$ & 1.74 (1.1 to 2.8$)$ & 0.03 \\
\hline$\geqslant 5$ & 2.71 (1.5 to 4.8$)$ & 0.0005 \\
\hline \multicolumn{3}{|l|}{ Type of hall } \\
\hline Mixed sex & Reference & \\
\hline Male only & $0.71(0.5$ to 1.0$)$ & 0.05 \\
\hline Female only & $0.52(0.3$ to 0.9$)$ & 0.03 \\
\hline Self catered & 0.73 (0.5 to 1.2$)$ & 0.2 \\
\hline \multicolumn{3}{|c|}{ Visited night club } \\
\hline No & Reference & \\
\hline Yes & $1.25(1.0$ to 1.6$)$ & 0.05 \\
\hline \multicolumn{3}{|c|}{ No of people kissed } \\
\hline 0 & Reference & \\
\hline 1 & $1.37(1.0$ to 1.8$)$ & 0.04 \\
\hline$\geqslant 2$ & 1.37 (0.9 to 2.1$)$ & 0.1 \\
\hline
\end{tabular}

Each risk adjusted for all other variables in table and antibiotic use in previous month.

Table 5 Typing data from 300 carriers in October 1997 and 333 strains acquired in first term in students previously negative for $N$ meningitidis

\begin{tabular}{lcc} 
Full typing details & No $(\%)$ of carriers & No (\%) of newly acquired strains \\
\hline C:2a:P1.5,2 (P1.5) & $3(1.0)$ & $21(6.3)$ \\
\hline W135:NT:P1.3,6 & $8(2.7)$ & $19(5.7)$ \\
\hline NG:NT:P1.16 & $7(2.3)$ & $17(5.1)$ \\
\hline NG:NT:NT & $16(5.3)$ & $11(3.3)$ \\
\hline NG:NT:P1.3,6 & $7(2.3)$ & $10(3.0)$ \\
\hline NG:NT:P1.5 & $16(5.3)$ & $5(1.5)$ \\
\hline B:NT:P1.15 & $10(3.3)$ & $6(1.8)$ \\
\hline B:NT:NT & $9(3.0)$ & $1(0.3)$ \\
\hline B:NT:P1.9 & $9(3.0)$ & $0(0)$ \\
\hline NG:NT:P1.15 & $9(3.0)$ & $5(1.5)$ \\
\hline $29 E: N T: P 1.5,2$ & $8(2.7)$ & $6(1.8)$ \\
\hline NG:15:P1.6 & $8(2.7)$ & $5(1.5)$ \\
\hline NG:4:NT & $7(2.3)$ & $3(0.9)$ \\
\hline$X: 21: P 1.16$ & $0(0)$ & $9(2.7)$ \\
\hline$Y: N T: P 1.5,2$ & $6(2.0)$ & $9(2.7)$ \\
\hline$B: 1: P 1.13$ & $0(0)$ & $7(2.1)$
\end{tabular}

$N G=$ not grouped; $N T=$ not typed. Group $C$ versus other strains $\chi^{2}=10.8$ (1df). $P=0.001$.

one person (KRN) with considerable experience. ${ }^{12}$ KRN also supervised the technique of the other operators. Furthermore, on each day during the first week, different operators assisted with swabbing in the morning and afternoon sessions yet there were no differences between morning and afternoon carriage rates on any day. We therefore believe that reliability was high between operators taking the swabs. The alternative explanation is that students who were more likely to be carrying meningococci on arrival at university were recruited later in the week. This seems unlikely as over $99 \%$ of students attended at their allotted time, and it seems unlikely that any systematic bias would have been introduced by choice of degree course. Furthermore, students are not allocated to halls of residence by course or faculty groups. The association of carriage with markers of social mixing also supports a causal link with acquisition after arrival at university.

Our main finding was a rapid increase in meningococcal carriage from $8 \%$ to $23 \%$ during the first week. Although the initial carriage rate was surprisingly low $(8 \%)$, this finding has now been replicated by a subsequent study performed in October 1999 with a different population of students, who had pharyngeal swabs taken both on arrival and one week later (data available on request). Therefore, we do not believe the initially low carriage rate to be artefactual and speculate that clearance of meningococci occurs during the summer holiday between leaving school and starting university. This may arise from dispersal of the sixth form group, resulting in lower rates of recolonisation.

We also noted that during the first week carriage was higher in catered halls. This agrees with a previous study, which identified an increased risk of invasive meningococcal disease at universities offering comparatively more accommodation in catered halls. We speculate that this may be due to fundamental differences in the physical structure and pattern of social interaction between catered and self catered halls at Nottingham University.

\section{Risk factors for carriage}

In our regression analysis, we identified active and passive smoking and intimate kissing as risk factors for carriage. These have been previously shown by other investigators. ${ }^{13}$ In addition, we noted that students living off campus were less likely to be carriers, which is also consistent with the theory of social mixing.

\section{Risk factors for acquisition}

Although all of the students in this analysis had an initial negative pharyngeal swab result, it is possible that some students were incorrectly identified as noncarriers during the first week of the study. This type of misclassification bias, inevitable in this type of study, will have had the effect of weakening any associations detected. For the same reason, our estimates of the prevalence of carriage should also be regarded as conservative. Nevertheless, we identified male sex, active smoking, visits to hall bars and nightclubs, intimate kissing, and mixed sex halls as risk factors for acquisition. Most of these factors have been previously identified in carriage and outbreak studies, ${ }^{14-16}$ but few have been addressed the risk of acquiring carriage. ${ }^{11}$ The lower rate of acquisition seen in female only halls probably reflects different patterns of social behaviour.

As expected, NG (not grouped) and serogroup B strains predominated in all swabs. Whereas carriage rates of serogroup $\mathrm{C}$ meningococci are significantly lower than for serogroup B or non-groupable 


\section{What is already known on this topic}

University students have been shown to be at greater risk of invasive meningococcal disease than other people of the same age

Meningococci have been shown to spread rapidly among military recruits and this is associated with increased rates of invasive disease

\section{What this study adds}

Meningococci spread rapidly among university students, probably due to social mixing

This explains the higher rates of invasive disease found among students each autumn during the first term of university and supports the recent introduction of meningococcal vaccination

meningococci, and typically less than $1 \%$ even in outbreaks, ${ }^{11417}$ our study found serogroup carriage rates of 3\% by December. This level increases the risk of outbreaks. Serogroup C disease is of particular importance as it is preventable by vaccine and has previously been linked to large clusters of disease among university students. ${ }^{56}$

The large comparative increase in the C:2a:P1.5 (P1.2) strains is noteworthy. This strain is known to be virulent and has been implicated in several major outbreaks $^{356}$; it represented $6.3 \%$ of all acquired strains in our study but only $1 \%$ of index strains identified in October. This illustrates the ability of highly virulent clones to transmit readily among students. Indeed the preferential transmission of this strain from a low baseline carriage rate may explain the 3-5 week delay usually observed between the start of university term and the peak incidence of cases and outbreaks.

During the beginning of university terms there is a rapid spread of meningococci in first year students, which is probably associated with social mixing, especially in catered halls. Our findings support the recent introduction of meningococcal vaccination for university students.

We thank Dr Jim Pearson for his advice on methods and statistics, Dr Angela White and her colleagues in the Cripps Health
Centre for use of facilities and general support, Keith Ashford for culturing the meningococci, and the Public Health Laboratory Service's meningococcal reference unit in Manchester for serogrouping, serotyping, and serosubtyping data.

Contributors: KRN initiated the study; he will act as guarantor for the paper. KRN, JSN-V-T, and DAAA'A supervised the study. KRN, JSN-V-T, NJ, KJ, RCBS, and RJM designed the study protocol. KRN and NJ analysed the data. All investigators contributed to the final paper.

Funding: Meningitis Research Foundation, Bristol.

Competing interests: None declared.

1 Hubert B, Caugant DA. Recent changes in meningococcal disease in Europe. Euro Surveillance 1997;2: 69-71.

2 Whalen CM, Hockin JC, Ryan A, Ashton F. The changing epidemiology of invasive meningococcal disease in Canada, 1985 through 1992. Emergence of a virulent clone of Neisseria meningitidis. JAMA 1995;273:390-4.

3 Jackson LA, Schuchat A, Reeves MW, Wenger JD. Serogroup C meningococcal outbreaks in the United States. An emerging threat. JAMA 1995;273:383-9.

4 Neal KR, Nguyen-Van-Tam JS, Monk P, O'Brien SJ, Stuart J, Ramsay M. Invasive meningococcal disease among university undergraduates: association with catered halls of residence. Epidemiol Infect 1999:122:351-8.

5 Anon. Outbreak of meningococcal disease in students in Cardiff. Commun Disease Rep 1996;6:425.

6 Anon. Clusters of meningococcal disease in university students. Commun Disease Rep 1997;7:393.

7 Jackson LA, Schuchat A, Gorsky RD, Wenger JD. Should college students be vaccinated against meningococcal disease? A cost-benefit analysis. Am J Public Health 1995;85:843-5.

8 Formic, an integrated questionnaire design and scanning package. wwwformic.co.uk. (Accessed 7 September 1999.)

9 Anderson J, Berthelson L, Jensen BB, Lind I. Dynamics of the meningococcal carrier state and characteristics of the carrier strains: a longitudinal study within three cohorts of military recruits. Epidemiol Infect 1998;121:85-94.

10 Devine LF, Pierce WE, Floyd TM, Rhode SL, Edwards EA, Siess EE, et al. Evaluation of group $\mathrm{C}$ meningococcal polysaccharide vaccine in marine recruits, San Diego, California. Am J Epidemiol 1970:92:25-32.

11 Melton LJ, Edwards EA, Devine EA, Devine LF. Differences between the sexes in the nasopharyngeal carriage of Neisseria meningitidis. Am J Epidemiol 1977;106:215-21.

12 Neal KR, Irwin DJ, Davies S, Kaczmarski EB, Wale MCJ. Sustained reduction in the carriage of Neisseria meningitidis as a result of a community meningococcal disease control programme. Epidemiol Infect 1998;121:487-93

13 Stuart JM, Cartwright KAV, Robinson PM, Noah ND. Effect of smoking on meningococcal carriage. Lancet 1989;ii:723-5.

14 Rønne T, Berthelesen L, Buhl LH, Lind I. Comparative studies on pharnygeal carriage of Neisseria meningitidis during a localized outbreak of serogroup C meningococcal disease. Scand J Infect Dis 1993;25:331-9.

15 Imrey PB, Jackson LA, Ludwinski PH, England III AC, Fella GA, Fox BC, et al. Outbreak of serogroup $\mathrm{C}$ meningococcal disease associated with campus bar patronage. Am J Epidemiol 1996;143:624-30.

16 Jelfs J, Jaluludin B, Munro R, Patel R, Kerr M, Daley D, et al. A cluster of meningococcal disease in western Sydney, Australia initially associated with a nightclub. Epidemiol Infect 1998;120:263-70.

17 Le Saux N, Ashton F, Rahman F, Ryan A, Ellis E, Tamblyn S, et al. Carriage of Neisseria species in communities with different rates of meningococcal disease. Can J Infect Dis 1992;3:60-4.

(Accepted 2 January 2000)

\section{A memorable patient \\ A surprising diagnosis}

The elderly woman was already frail when she came to me complaining of a cough and weight loss. Alarm bells rang as I examined her and arranged for blood tests and a chest $x$ ray examination. As I feared, the chest radiograph was suspicious, and referral was advised.

The chest physician concluded that bronchial carcinoma was almost certain and recommended awaiting the outcome of events, rather than subjecting her to an unpleasant bronchoscopy. Rather to my surprise neither she nor her family agreed, and she came back to me insisting on further investigation.

Some time later I rather hesitantly explained the eventual diagnosis of tuberculosis to her and was astonished by her calm response. "I rather thought it might be that, doctor," she said, "after that trouble I had years ago. Didn't I ever tell you I'd had tuberculosis as a child?" Red faces all round, but a well, elderly patient five years later

Catherine Harkness general practitioner, Willey, Warwickshire

We welcome articles of up to 600 words on topics such as A memorable patient, A paper that changed my practice, My most unfortunate mistake, or any other piece conveying instruction, pathos, or humour. If possible the article should be supplied on a disk. Permission is needed from the patient or a relative if an identifiable patient is referred to. We also welcome contributions for "Endpieces," consisting of quotations of up to 80 words (but most are considerably shorter) from any source, ancient or modern, which have appealed to the reader. 